\title{
Numerical and experimental study of impulsive sound attenuation of an earmuff
}

\author{
Felipe Vergara $^{\mathrm{a}}$, Samir N. Gerges ${ }^{\mathrm{a}, *}$ and Robert S. Birch ${ }^{\mathrm{b}}$ \\ ${ }^{a}$ Federal University of Santa Catarina, Department of Mechanical Engineering, Laboratory of Acoustics and \\ Vibrations, Post Box 476, Florianópolis, Santa Catarina, Brazil \\ E-mail: zeta@emc.ufsc.br; samir@emc.ufsc.br \\ ${ }^{\mathrm{b}}$ University of Liverpool, Impact Research Centre, Department of Engineering, Brownlow Hill, Liverpool, L69 \\ $3 G H, U K$ \\ E-mail: rsb123@liv.ac.uk
}

\begin{abstract}
This work compares the results of laboratory experiments with numerical modelling using the finite element method in order to assess the attenuation of hearing protectors under conditions of high amplitude impulse noise. Comparative data for the finite element simulation was provided from a series of experiments using a shock tube, acoustic test fixture, ear canal simulator and partial head form. The numerical model comprised a finite element mesh of fluid and porous materials in order to model the earmuff hearing protector coupled to the auditory canal. The results show that a simple 2-D finite element model is capable of making a reasonable prediction of the attenuation of an earmuff provided that headband force is also included in the model.
\end{abstract}

\section{Introduction}

Earmuff type hearing protectors offer a quick method for hearing conservation. In order to determine the acoustic performance of a hearing protector when subjected to impulsive noise, knowledge of any nonlinearity in the muff response to peak level, rise time and duration of the impulse is required. The evaluation of hearing protectors for the attenuation of high amplitude impulsive noise cannot be carried out using the conventional subjective real ear attenuation at threshold (REAT) technique [7]. Safety, resolution, cost and turn-around-time are inherent problems in the REAT method which often lead to a design synthesis based on the manufacture and test of several prototypes. In particular, REAT cannot be carried out at high noise levels. Quicker and less expensive techniques for the development of protectors are desirable. The use and application of the finite element method (FEM), for example, offers a method of design and development. Also, the reproduction in laboratory of impulse noises whose

\footnotetext{
*Corresponding author.
}

characteristics are similar to real sources is needed to help the development of the FEM approach. The objective of the work here presented is to compare the performance of an earmuff HPD (hearing protection device) measured using a shock tube, impulse generator, acoustic test fixture (ATF) and ear canal simulator and a numerical model using FEM. The shock tube is capable of producing repeatable pressure (noise) impulses with characteristics similar to those experienced from real sources.

\subsection{Attenuation of hearing protectors}

The external ear comprises the pinna, auditory canal and tympanum. An ear canal length between $27-37 \mathrm{~mm}$ and diameter of $7.5 \mathrm{~mm}$ at the tympanum are approximate dimensions for the average adult. The walls of the auditory canal have a dilation impedance approximately four orders of magnitude greater than the air in canal itself and can be treated as a rigid surface [8].

The passive earmuff may be regarded as a low pass filter against impulsive noise. When the impulse pressure wave incidens on the protector, the earmuff may 


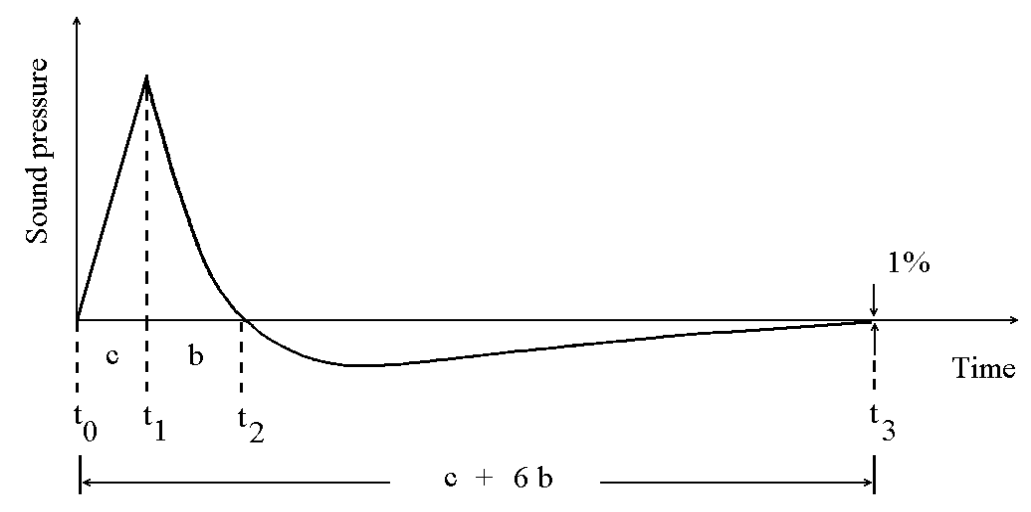

Fig. 1. Friedlander pulse.

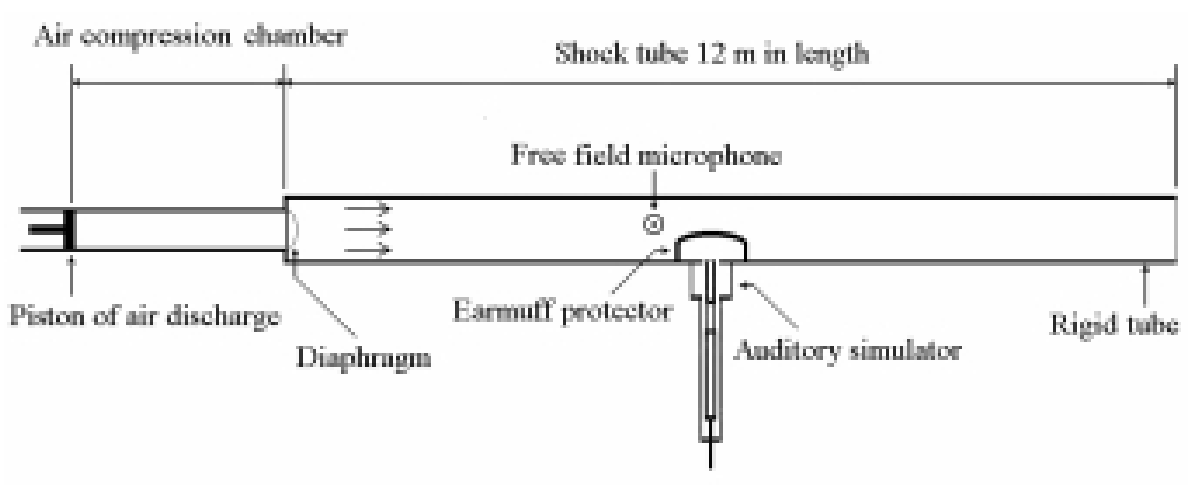

Fig. 2. Shock wave tube system.

act with a global damped oscillatory motion against the head with a resonant frequency in the range 100$500 \mathrm{~Hz}$ [5]. The attenuation of a hearing protector can be expressed as:

$$
\text { Attenuation }=20 \log \left(\frac{p_{\text {freefield }}}{p_{\text {with }}}\right)
$$

where $p_{\text {freefield }}$ and $p_{\text {with }}$ are the sound pressures in the free field (outside the muff) and at the tympanum position (with an earmuff), respectively. Some authors consider the reduction to be the insertion loss, the ratio of the pressures at the tympanum with and without a muff protector fitted [4].

\subsection{Physical factors of impulsive noise}

The impulsive transient noise is generated by a fast discharge of energy (e.g., electrical or chemical) into the atmosphere. Impulsive noise is particularly dangerous to hearing as it is the inability of the human auditory system to sense the peak level accurately in relation to the levels of steady state noise that can allow damage to occur. The physical characteristics of an impulse are dependent on the geometry, of the scale of the source and of the atmosphere where it may propagate. The most significant physical parameters of a noise impulse are normally considered to be:

i) Peak pressure level or maximum value of sound pressure (usually expressed in $\mathrm{dB}$, using a reference pressure of $20 \mu \mathrm{Pa}$ ).

ii) Rise time for the sound pressure to reach the maximum peak pressure.

iii) Duration of the main pressure pulse (there are several definitions for this).

The instantaneous pulse of energy of a point source in the free field, that is, in a field without reflecting surfaces, produces a pressure pulse with characteristics known as a Friedlander pulse or wave [7]. The difference between times $t_{1}$ and $t_{0}=0$ in the Fig. 1 defines the rise time of the peak sound pressure level at time $t_{1}$. The time taken by the pressure to fall to zero at $t_{2}$ defines another parameter sometimes referred to as Duration A and corresponds to the difference between 


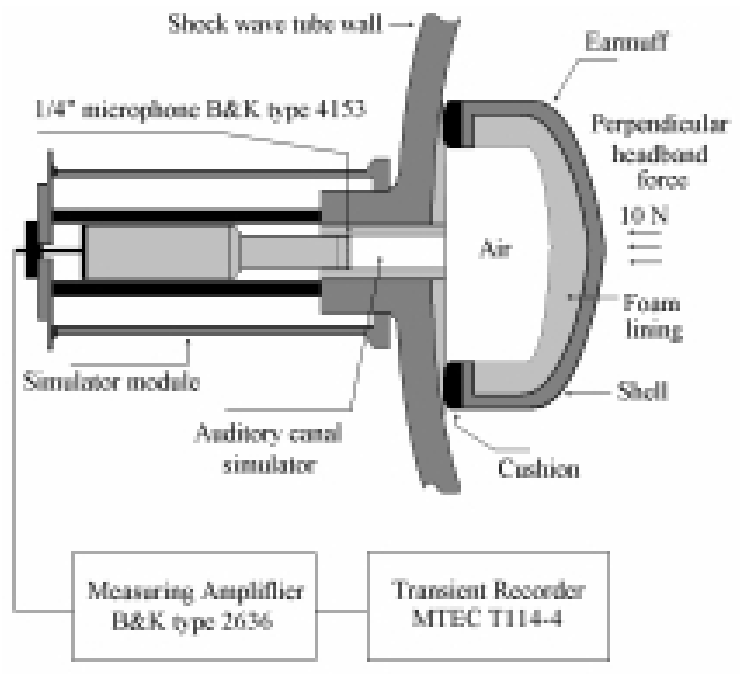

Fig. 3. The acoustics test fixture module used to measure the sound attenuation of hearing protectors.

$t_{2}$ and $t_{0}$. The final part of the pulse comprises a period of rarefaction that extends until $t_{3}$ (approx. $\mathrm{c}+6 \mathrm{~b}$ ) where the pressure returns to ambient state again. In practice this is considered to be less than $1 \%$ of the peak level [5].

\section{Experimental measurements using the shock wave tube}

Noises with impulsive characteristics were produced in a shock wave tube using the rapid discharge of compressed air to generate an impulsive pressure pulse. This was achieved using a small pressure chamber and rupturing diaphragm located at one end of the shock tube as illustrated in the Fig. 2. The shock tube was manufactured using $150 \mathrm{~mm}$ diameter rigid plastic tubing of $12 \mathrm{~m}$ in length.

In order to assess hearing protectors under conditions of high amplitude impulse noise an ATF was constructed comprising an ear canal simulator module and partial head form. The ATF module was used to examine the performance of a shell type protector with a wave pulse passing in a grazing type test. By taking simultaneous measurements from the external field and the occluded canal simulator, the attenuation of the earmuff can be estimated. Flanking transmission of sound to the ATF was minimised by using a double sealed construction to house the auditory simulator and instrumentation (see Fig. 3). Each auditory canal simulator itself comprised an $8 \mathrm{~mm}$ diameter plastic tube and $\frac{1}{4}^{\prime \prime}$
B\&K type 4135 microphone. Signals from the ATF modules were processed using B\&K type 2636 measuring amplifiers and recorded on the MTEC T114-4 transient recorder. A series of tests considered an earmuff protector with a 54 gram mass, an internal volume of $209 \mathrm{ml}$ and high density foam lining reducing the volume to $137 \mathrm{ml}$, approximately. The shell of the muff was manufactured from rigid plastic and had soft plastic/foam head seal with virtually linear stiffness characteristics. During testing a normal clamping force of $10 \mathrm{~N}$ was applied to the muff shell to ensure a good seal and representative condition.

\section{Numerical simulation applying finite element method (FEM)}

The mathematical formulation for the determination of the sound pressure in transient acoustic problems uses the FEM and the direct time integration procedure to solve the linear wave equation that governs the small amplitude propagation of acoustic waves within a homogeneous acoustic medium. Considering the variations of acoustic pressure as the main variable the wave equation is:

$$
\nabla^{2} p(x, t)-\frac{1}{c^{2}} \frac{\partial^{2} p(x, t)}{\partial t^{2}}=0 \quad t \geqslant 0
$$

Using the wave equation with the boundary conditions (sound pressure and impedance), the variational form of Eq. (2), the discretization of the domain into finite elements and the selection of the adequate shape functions a system of linear differential equations with constant coefficients is obtained [3]:

$$
[M]\{\ddot{P}\}+[C]\{\dot{P}\}+[K]\{P\}=\left\{F_{A}\right\}
$$

Where $F_{A}$ is the vector of nodal acoustics forces. The stiffness $K$, damping $C$ and mass $M$ matrices are computed once only and they are independent of the time step.

The parameters that control the numerical integration in the time domain follow the outline the procedure of Newmark integration that considers $\alpha=0.25$, and $\delta=0.5$. With these values of $\alpha$ and $\delta$ the transient solution does not exhibit an amplitude error. To avoid oscillation or overshoot effects a mesh refinement and step selection was used such that the product of the speed of the sound by the step in the time, $c x \Delta t$, would be equal to element size. The finite element procedure was carried out controlling the Newmark parameters and choosing an appropriate time step $(343 \mathrm{~m} / \mathrm{s} \times$ $80.5 \mu \mathrm{s}=2.8 \mathrm{~mm})[2,3]$. 


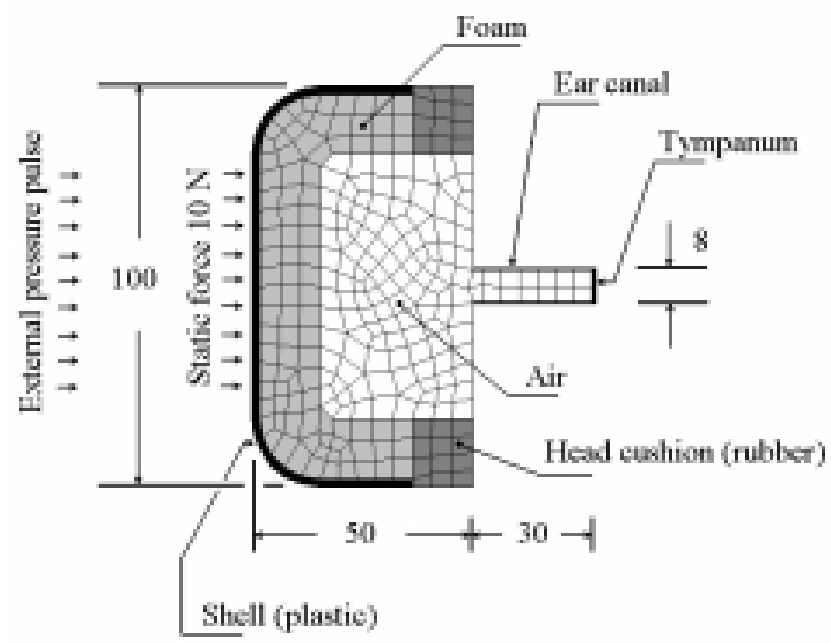

Fig. 4. Finite element model of the auditory canal and earmuff protector.

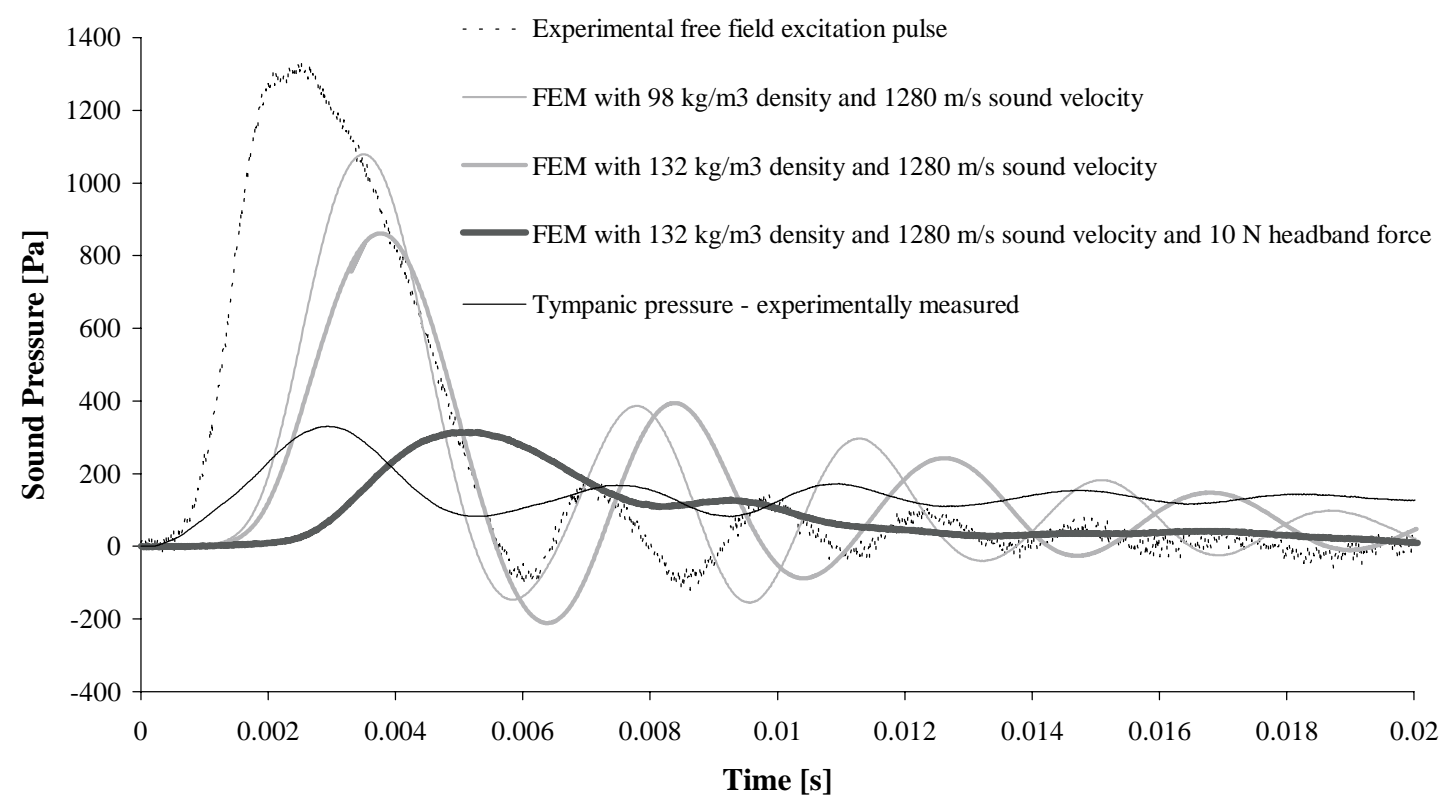

Fig. 5. Test case 1 - pressure vs. time histories for the free field excitation pulse and experimental and FEM predicted tympanic pressures.

The test data provided by the shock tube experiments were compared with the results from the FEM simulation. FEM modelling was carried out using the Sysnoise 5.4 software with the time domain module of linear wave propagation [9]. The model comprised a finite element mesh of fluid type elements (FLUID29 from ANSYS 5.3 software) to model the earmuff hearing protector coupled to the auditory canal as a 2-D model. FLUID29 is used to model problems in a fluid medium and in the fluid-structure interface. This element is rectangular and has four corner nodes with three degrees of freedom per node: translations in the nodal $x$ and $y$ directions and pressure. Also, the element can include damping and absorbing material at the interface and can be used into transient analysis. These analyses are limited to small acoustics pressures, i.e., the changes in density are small compared with the mean density [1]. Excitation of the FEM was achieved using the experimentally generated external (free field) pressure versus time pulse (see Fig. 5) rather than the idealised Friedlander function shown in Fig. 1.

The protector and the ear canal were modelled as two 


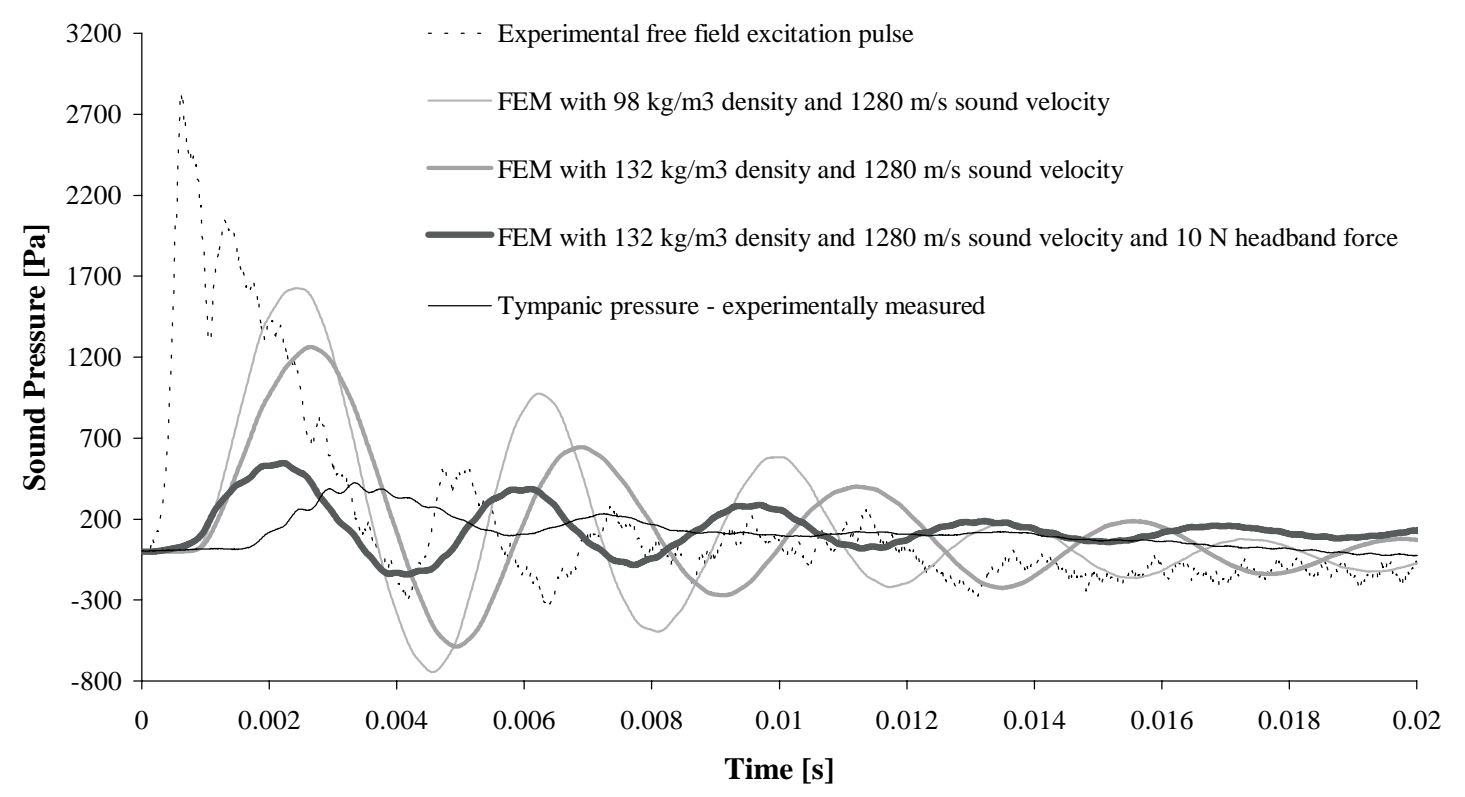

Fig. 6. Test case 2 - pressure vs. time histories for the free field excitation pulse and experimental and FEM predicted tympanic pressures.

dimensional rectangular areas of $50 \mathrm{~mm} \times 100 \mathrm{~mm}$ and $30 \mathrm{~mm} \times 8 \mathrm{~mm}$, respectively. Boundary conditions for the earmuff-canal model were imposed at the protector surfaces and at the ends of the canal using impedances for the tympanum. Material properties for the plastic and foam construction of the canal and earmuff protector shown in Fig. 4 were taken as follows:

i) The protector shell is defined as a solid material of $1200 \mathrm{~kg} / \mathrm{m}^{3}$ density and with a $1500 \mathrm{~m} / \mathrm{s}$ sound velocity with $2 \mathrm{~mm}$ thickness;

ii) The air cavity was treated as a homogeneous medium $\left(1.21 \mathrm{~kg} / \mathrm{m}^{3}\right.$ density and $343 \mathrm{~m} / \mathrm{s}$ sound velocity) and

iii) An impedance value of $10^{8} \mathrm{Ns} / \mathrm{m}^{5}$ was imposed at the position representing the tympanum, while a value of $10^{4} \mathrm{Ns} / \mathrm{m}^{5}$ was used to represent the impedance of the walls of the auditory canal [6].

iv) The density value for the foam liner was taken as $132 \mathrm{~kg} / \mathrm{m}^{3}$ with a sound speed of $1280 \mathrm{~m} / \mathrm{s}$, but the FEM model also considered a value $98 \mathrm{~kg} / \mathrm{m}^{3}$ and $1280 \mathrm{~m} / \mathrm{s}$.

v) The headband force of $10 \mathrm{~N}$ was imposed as an evenly distributed line on the outside of the earmuff shell, but the FEM also considered zero headband force.

vi) Material damping was not included in the model.

\section{Discussion}

Pressure versus time history recordings from two experimental test cases are presented in Figs 5 and 6 where it can be seen that the free field pulse excites a delayed pressure response at the simulated tympanum position (occluded by the HPD). Similarly, the predictions from the FEM model are superimposed on Figs 5 and 6 and summaries of these results are presented in Tables 1 and 2 .

It can be seen from Figs 5 and 6 that, while keeping all other earmuff material properties constant, the predictions of the FEM model exhibit a strong sensitivity to the density of the foam rubber liner. In particular, the attenuation of the initial peak of the pressure pulse can be seen to be greatly influenced by liner density. Also, the initial amplitude of the free field excitation produces significant changes in the attenuation of the tympanum pressure in both the experiment and FEM model. The combination of low density foam liner and relatively large rise time of free field pulse appears to result in the FEM predicting a smaller attenuation of the peak when compared to the free field pulse with a shorter rise time (see Figs 5 and 6 also Tables 1 and 2 ). The inclusion of the headband force as a line load across the shell improves the prediction for the attenuation of the peak and subsequent response in the time domain.

The time lag between the initial excitation of the free field pulse and response of the tympanum is also 
Table 1

Comparison of measured tympanum pressure to those predicted by the FEM model using a $156.5 \mathrm{~dB}$ peak, $1 \mathrm{~ms}$ rise time free field pulse (Test case 1)

\begin{tabular}{llcccccc}
\hline Location & Type & $\begin{array}{c}\text { HPD liner } \\
\text { sound speed } \\
{[\mathrm{m} / \mathrm{s}]}\end{array}$ & $\begin{array}{c}\text { HPD liner } \\
\text { density } \\
{\left[\mathrm{kg} / \mathrm{m}^{3}\right]}\end{array}$ & $\begin{array}{c}\text { Peak } \\
\text { pressure } \\
{[\mathrm{kPa}]}\end{array}$ & $\begin{array}{c}\text { Peak SPL } \\
{[\mathrm{dB}]}\end{array}$ & $\begin{array}{c}\text { Attenuation } \\
{[\mathrm{dB}]}\end{array}$ & $\begin{array}{c}\text { Rise time } \\
{[\mathrm{ms}]}\end{array}$ \\
\hline Free field & Experimental pulse & - & - & 1.33 & 156.5 & - \\
Tympanum & Experimental & 1280 & 320 & 0.31 & 143.9 & 12.6 & 1.71 \\
& FEM prediction & 1280 & 98 & 1.08 & 15.6 & 1.9 & 1.32 \\
& FEM prediction & 1280 & 320 & 0.86 & 152.7 & 3.8 \\
& FEM prediction (10 N force) & 1280 & 320 & 0.33 & 144.4 & 1.57 \\
\hline
\end{tabular}

Table 2

Comparison of measured tympanum pressure to those predicted by the FEM model using a $163 \mathrm{~dB}$ peak and 0,24 ms rise time free field pulse (Test case 2)

\begin{tabular}{llcccccc}
\hline Location & Type & $\begin{array}{c}\text { HPD liner } \\
\text { sound speed } \\
{[\mathrm{m} / \mathrm{s}]}\end{array}$ & $\begin{array}{c}\text { HPD liner } \\
\text { density } \\
{\left[\mathrm{kg} / \mathrm{m}^{3}\right]}\end{array}$ & $\begin{array}{c}\text { Peak } \\
\text { pressure } \\
{[\mathrm{kPa}]}\end{array}$ & $\begin{array}{c}\text { Peak SPL } \\
{[\mathrm{dB}]}\end{array}$ & $\begin{array}{c}\text { Attenuation } \\
{[\mathrm{dB}]}\end{array}$ & $\begin{array}{c}\text { Rise time } \\
{[\mathrm{ms}]}\end{array}$ \\
\hline Free field & Experimental pulse & - & - & 2.84 & 163.0 & - & 0.24 \\
Tympanum & Experimental & 1280 & 320 & 0.414 & 146.3 & 16.7 & 1.30 \\
& FEM prediction & 1280 & 98 & 1.624 & 158.2 & 4.8 & 1.07 \\
& FEM prediction & 1280 & 320 & 1.257 & 155.9 & 7.0 & 1.30 \\
& FEM prediction (10 N force) & 1280 & 320 & 0.546 & 148.7 & 14.3 & 1.00 \\
\hline
\end{tabular}

reflected in the FEM predictions and is $25 \%$ to $40 \%$, approximately, smaller than the experimental case (see Figs 5 and 6). Unlike the peak pressure response, this time lag appears to be less sensitive to changes in the liner density, but is sensitive to the inclusion of headband force.

The FEM was found to be considerably less sensitivity to the other material parameters, such as: porosity, flow resistivity and structural factor. Improvements in the model could consider the boundary condition at the shell/air interface where the pulse and headband forcing pulse acts, plus material damping effects. Clearly, the geometrical characteristics of the muff shell are greatly simplified using 2-D model and a more refined model might consider the effects of the headband contact and local changes in thickness of the shell wall as well as the 3-D case.

The global vibration of the earmuff shell is not considered in the current FEM, but is a significant consideration when it is excited by a high amplitude pulse of relatively long duration. Effectively, the shell and head cushion form a spring-mass-damper system making the overall shell appear less rigid to the free field pulse. Although this has the effect of increasing acoustic energy absorption into the earmuff (cushion), global motion of the shell can produce dynamic compression of the air space inside the muff increasing, potentially, the tympanic pressure.

Finally, considering the simple form of ATF used in this work, only an idealised response of a hearing protector can be obtained experimentally. In reality, the bone transmission, geometrical variation of the human pinna and ear canal and the effect of wearing and fitting conditions are also variables that eventually would need to be considered if FEM were to represent the human case.

\section{Conclusions}

A simple 2-D finite element model was used to examine the performance of an earmuff subject to high amplitude short duration pressure pulses (noise). Density of the foam liner and inclusion of the headband force were considered as variables in the model. A comparison with experimental data, obtained from shock tube tests, showed the FEM model to be capable of making a reasonable prediction for the attenuation of the free field pulse at the tympanum position when the headband force was included. The FEM model appeared to be particularly sensitive to changes in density of the foam liner in the earmuff shell structure while being relatively insensitive to changes in other material parameters porosity, flow resistivity and structural factor.

\section{Acknowledgements}

The authors wish to acknowledge the support of the Conselho Nacional de Desenvolvimento Cien- 
tifico e Tecnológico (CNPq) of Brazil, process number 300619/98-7 and 520165/96-NV, for their support of this project.

\section{References}

[1] ANSYS Release 5.3 User's manual, 1994.

[2] J.P. Coyette and I.P. Segaert, Investigation of acoustic wave propagation in a 2D rectangular tube, LMS Numerical Technologies NV, Belgium, 1997.

[3] J.P. Coyette, Application of finite element and boundary element models to transient acoustic problem, IX International Conference on Vehicle Structural Mechanics and CAE, Michigan, USA, April 4-6, 1995.

[4] E. Osmundsen and K. Gjavenes, The noise attenuation of weak shock waves by ear muffs, Journal of Sound and Vibration 77(3) (1981), 375-385.

[5] D. Smeatham and P.D. Wheeler, On the performance of hearing protectors in impulsive noise, Applied Acoustics 54(20) (1998), $165-181$.

[6] F. Vergara, Modelagem numérica e medições acústicas de protetores auditivos para rúdos impulsivos, Master Dissertation, Federal University of Santa Catarina, Florianópolis, Brazil, 1999.

[7] G.F. Smoorenburg, Assessment of hearing protector performance in impulsive noise, Final Report, TNO Human Factors Research Institute, Netherlands, 1996.

[8] R. Rabbit and M. Holmes, Three-dimensional acoustics waves in the ear canal and their interaction with the tympanic membrane, Journal Acoustics Society of America 83(30) (1988), 1064-1080.

[9] SYSNOISE Revision 5.4 Users manual, 1999. 

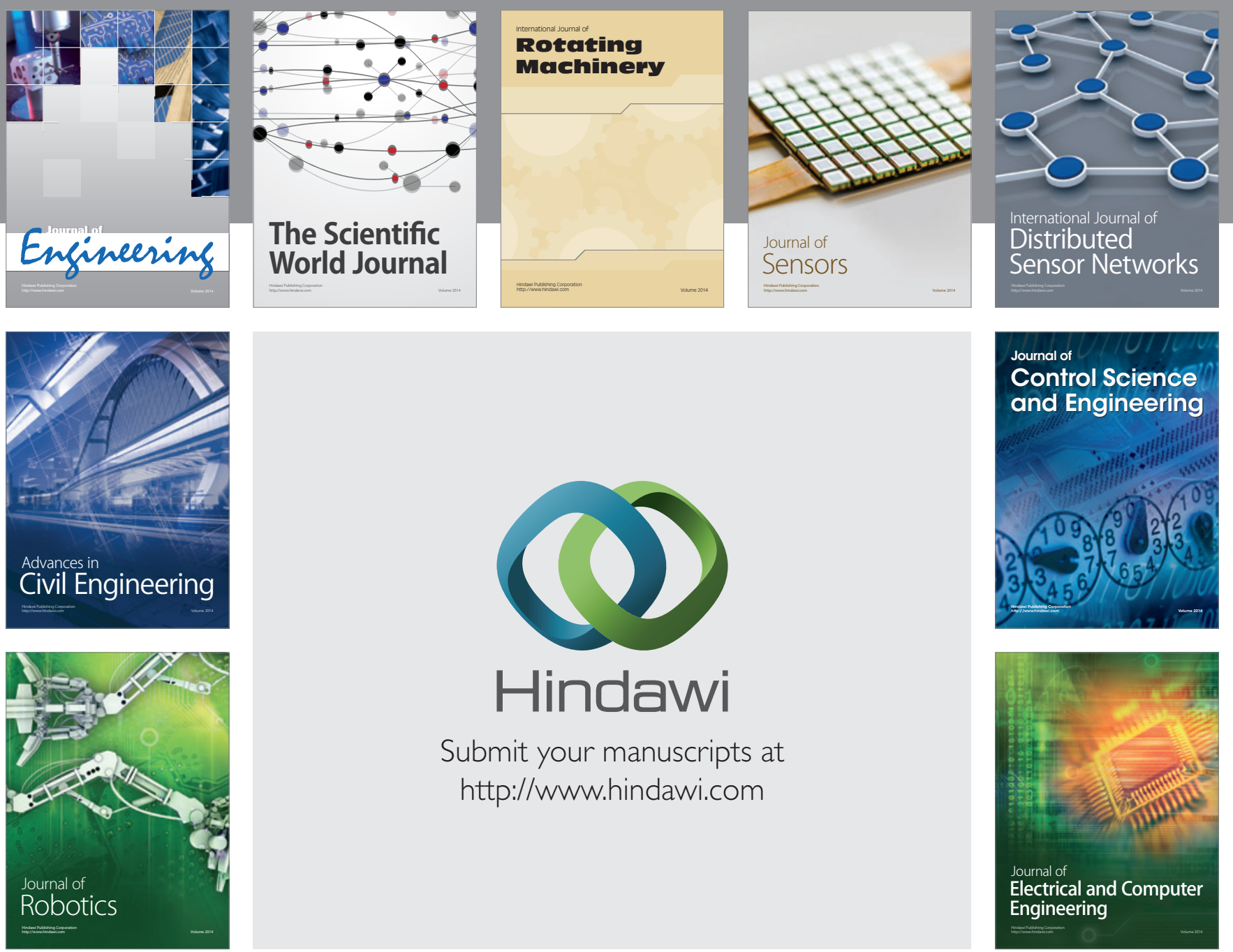

Submit your manuscripts at

http://www.hindawi.com
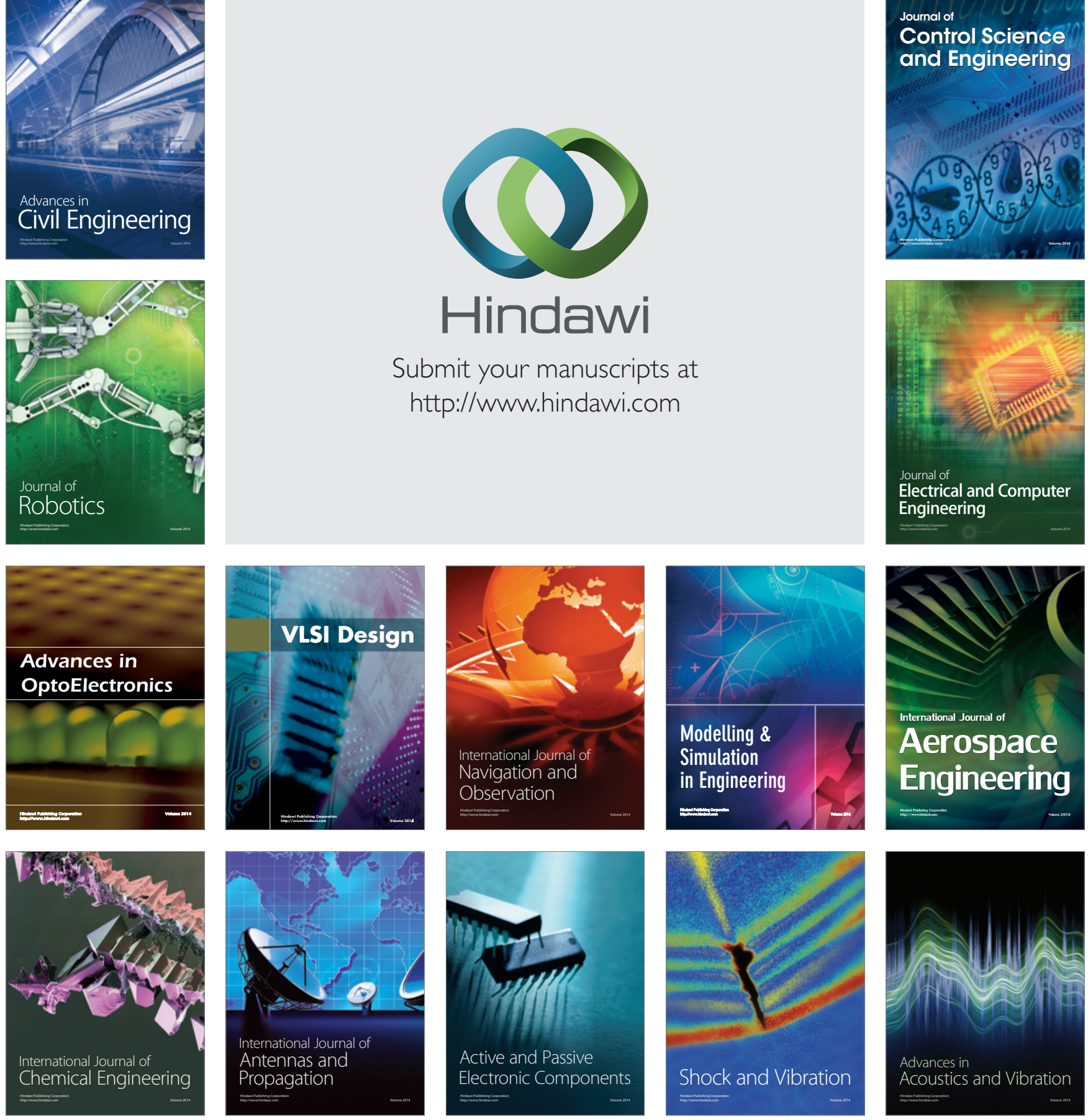